\title{
Efeito de Diferentes Tipos de Argilas e Modificadores Orgânicos na Morfologia e Propriedades Térmicas dos Nanocompósitos de PET
}

\author{
Itamara Farias Leite \\ Programa de Pós-Graduação em Ciência de Materiais, UFPE
}

Oscar Loureiro Malta

Departamento de Química Fundamental, UFPE

Claudia M. 0. Raposo

Unidade Acadêmica de Mineração e Geologia, UFCG

\author{
Luís Eduardo Canedo, Laura H. de Carvalho, Suédina M. de L. Silva \\ Unidade Acadêmica de Engenharia de Materiais, UFCG
}

\begin{abstract}
Resumo: Argilas bentoníticas natural e purificada (AN e AP) e montmorilonita (MMT), modificadas organicamente com os sais alquil amônio (A), alquil fosfônio (F) e com uma mistura de ambos (A e F), foram incorporadas, em teor de $1 \%$ em massa, ao PET pelo processo de fusão. Os híbridos obtidos foram caracterizados por difratometria de raios X (DRX), termogravimetria (TG), calorimetria exploratória diferencial (DSC) e microscopia eletrônica de transmissão (MET). Nanocompósitos com morfologia intercalada ordenada e intercalada desordenada/esfoliada foram obtidos quando manufaturados com as argilas organofilizadas com sal amônio e com a mistura dos sais amônio e fosfônio. A incorporação das argilas organofilizadas com o sal fosfônio ao PET não resultou na formação de nanocompósitos. As argilas organofílicas ANOA, APOF e MMTOF, quando misturadas ao PET, provocaram aumento da sua estabilidade térmica, além de atuarem como agentes nucleantes heterogêneos para o referido polímero, aumentando sua temperatura de cristalização. Este resultado é significativo do ponto de vista industrial, pois a baixa velocidade de cristalização do PET dificulta seu uso na preparação de artefatos injetados. Além disso, como as argilas bentoníticas fornecidas por empresa local apresentaram comportamento semelhante ao da montmorilonita importada, o uso dessa matéria-prima de menor custo, modificada com tecnologia nacional, pode ser uma alternativa atraente para aplicações do PET moldado por injeção onde o custo é um fator primordial.
\end{abstract}

Palavras-chave: Argila, surfactante, PET, morfologia, propriedades térmicas, nanocompósitos.

\section{Effect of Different Types of Clays and Organic Modifiers on the Morphology and Thermal Properties of PET Nanocomposites}

Abstract: Montmorillonite (MMT), natural (AN) and purified (AP) bentonite clays modified with alkyl ammonium (A), alkyl phosphonium (F) and a mixture of both (A and F) salts were incorporated (1\% w/w) into PET by melt blending. The hybrids thus obtained were characterized by XRD, TG, DSC and TEM. Nanocomposites having intercalated ordered structures and intercalated disordered/exfoliated structures were obtained with the clays modified with the ammonium and the mixture of ammonium and phosphonium salts, respectively. A nanocomposite was not obtained with the addition of $1 \% \mathrm{w} / \mathrm{w}$ of an alkyl phosphonium modified clay into PET. The organoclays ANOA, APOF and MMTOF, when added to PET not only led to an increase in its thermal stability but also acted as heterogeneous nucleating agents, increasing its crystallization temperature. This result is industrially significant as the low crystallization rate of PET makes its use difficult in preparing injected goods. Besides, as the behaviour of the bentonite clays supplied by a local industry was similar to that of the imported montmorillonite, the use of this raw material of lower cost, modified with national technology, can be an attractive technology for injection molded PET applications, where cost is of utmost importance.

Keywords: Clay, surfactant, PET, morfology, thermal properties, nanocomposites.

\section{Introdução}

A síntese de novos materiais com desempenho e propriedades otimizadas constitui uma área em constante expansão em Ciência de Materiais. Um avanço significativo nesta área tem ocorrido com a síntese de nanocompósitos, onde a ordem estrutural dentro do material pode ser controlada em escala nanométrica ${ }^{[1]}$.

Um dos sistemas mais promissores é o híbrido baseado em polímero orgânico e argilomineral constituído de silicatos em camadas $^{[2-10]}$. Dentre os vários silicatos em camadas, as montmorilonitas têm sido uma das mais empregadas, nos últimos anos, como carga para a preparação de nanocompósitos poliméricos $^{[11-14]}$. Além da montmorilonita, a bentonita também vem sendo empregada e investigada para tal finalidade ${ }^{[15-17]}$. Contudo, um dos grandes problemas associado com o uso desta é a presença usual de impurezas não argilosas como quartzo, feldspato, gipsita, albita, halita, calcita, pirita, carbonatos de sódio e outros ${ }^{[18-19]}$, e de matéria orgânica como impureza intrínseca ${ }^{[18,20]}$. Portanto, tratamento de purificação vem sendo empregado em bentonitas como uma etapa necessária e de grande importância

Autor para correspondência: Suédina Maria de L. Silva, Unidade Acadêmica de Engenharia de Materiais, CCT, Universidade Federal de Campina Grande, Rua Aprígio Veloso, 882, Bodocongó, CEP 58429-900, Campina Grande, PB, Brasil. E-mail: suedina@dema.ufcg.edu.br 
para garantir reprodutibilidade nos resultados ${ }^{[17,19]} \mathrm{e}$, assim poder ser usada com sucesso na preparação de nanocompósitos poliméricos.

Devido à natureza inorgânica e hidrofílica das argilas, a esfoliação das mesmas em matrizes poliméricas orgânicas pode ser limitada. Por esta razão, a preparação de nanocompósitos geralmente necessita de uma modificação orgânica da argila para torná-la hidrofóbica e com caráter orgânico. A intercalação de cátions orgânicos nas galerias da argila não somente aumenta o espaçamento interlamelar, como também diminui as interações entre as lamelas adjacentes da argila e facilita a subsequente penetração do polímero entre as camadas da mesma ${ }^{[21-24]}$.

Os modificadores orgânicos à base de alquil amônio têm sido tradicionalmente empregados na modificação orgânica de argilas. Contudo, a degradação térmica das argilas organofilizadas com este tipo de modificador (surfactante) inicia em temperaturas próximas de $200{ }^{\circ} \mathrm{C}$, que corresponde à faixa de temperatura de processamento da maioria dos polímeros termoplásticos ${ }^{[25]}$. Embora os modificadores alquil amônio tenham sido empregados com sucesso na preparação de nanocompósitos poliméricos, sua decomposição pode alterar a interface polímero/argila, dificultando a obtenção de nanocompósitos ${ }^{[26]}$.

Segundo Shah \& Paul (2006) ${ }^{[27]}$ a degradação da argila organofílica pode limitar a extensão da intercalação e/ou esfoliação nos nanocompósitos. Então, para superar esta limitação, Leite et al. (2010 $)^{[17]}$ mostraram que a estabilidade térmica das argilas modificadas com o sal alquil amônio pode significativamente ser melhorada pela aplicação de procedimentos de purificação.

Vários modificadores orgânicos estáveis termicamente, tais como alquil pirídinio ${ }^{[28]}$, alquil fosfônio ${ }^{[29]}$ e alquil imidazólio ${ }^{[30]}$ têm sido propostos como rotas alternativas na obtenção de argilas organofílicas estáveis termicamente e potencialmente úteis para a obtenção de nanocompósitos usando matrizes poliméricas que requerem altas temperaturas no processamento ${ }^{[24,29]}$. Entretanto, além de estabilidade térmica, as argilas organofílicas devem ainda possuir afinidade química com o polímero, para assim favorecer a formação de nanocompósitos poliméricos. Portanto, este trabalho tem como finalidade avaliar o efeito de diferentes tipos de argilas organofílicas e modificadores orgânicos na morfologia e propriedades térmicas dos nanocompósitos de PET.

\section{Experimental}

\section{Materiais}

O poli(tereftalato de etileno) (PET), BG1180-W, grau garrafa, fornecido pela Braskem/BA sob a forma de pellets de coloração branca foi usado como matriz polimérica para a preparação dos nanocompósitos. De acordo com as informações do fornecedor, este polímero apresenta uma viscosidade intrínseca de $0,80 \pm 0,02 \mathrm{dL} \cdot \mathrm{g}^{-1}$; teor de acetaldeído equivalente a $1.0 \mathrm{ppm}$; temperatura de fusão cristalina de $247 \pm 5^{\circ} \mathrm{C}$ e densidade na faixa de $1,39-1,41 \mathrm{~g} . \mathrm{cm}^{-3}$.

A bentonita Argel 35 (AN) com capacidade de troca catiônica (CTC) desta bentonita foi de $92 \mathrm{meq} / 100 \mathrm{~g}$ de argila, conforme método descrito por Phelps \& Harris (1968) ${ }^{[31]}$, foi fornecida pela Bentonit União Nordeste (BUN-Campina Grande, Brasil). Esta argila foi purificada, visando à remoção de matéria orgânica da mesma, de acordo com o procedimento reportado previamente ${ }^{[32]}$ e codificada como AP. Para fins comparativos utilizou-se uma montmorilonita sódica comercial - Cloisite ${ }^{\circledR} \mathrm{Na}^{+}$(MMT), fornecida pela Southern Clay Products/EUA com uma CTC de 92 meq/100 g de argila. Os sais orgânicos brometo de hexadecil trimetil amônio, $\mathrm{C}_{16} \mathrm{H}_{33}\left(\mathrm{CH}_{3}\right)_{3} \mathrm{NBr}$, (A; massa molar de 364,45 g. $\mathrm{mol}^{-1}$ ) e brometo de hexadecil tributil fosfônio, $\mathrm{C}_{16} \mathrm{H}_{33}\left(\mathrm{C}_{4} \mathrm{H}_{9}\right)_{3} \mathrm{PBr}$, (F; massa molar de 507,65 g. mol ${ }^{-1}$ ), fornecidos pela Vetec e Aldrich, respectivamente, foram usados como recebidos na modificação orgânica das argilas.

\section{Métodos}

As bentonitas, natural (AN) e purificada (AP), bem como a montmorilonita sódica comercial (MMT) foram modificadas organicamente, por reação de troca iônica, com os sais orgânicos alquil amônio (A) e alquil fosfônio (F). A bentonita purificada (AP) foi também modificada com a mistura de ambos os sais A e F. Dispersões aquosas de $2 \%$ de argila foram aquecidas a aproximadamente $70 \pm 5^{\circ} \mathrm{C}$. A estas dispersões foram adicionadas $100 \%$ do sal A, do sal F e da mistura de ambos os sais A e F em quantidades equivalentes à capacidade total de troca catiônica da argila. As dispersões foram mantidas sob agitação mecânica por 30 minutos a $3000 \mathrm{rpm}$. Passado este tempo, permaneceram em repouso por 24 horas à temperatura ambiente, sendo então, filtradas, lavadas e secas a $60 \pm 5{ }^{\circ} \mathrm{C}$ por um período de 48 horas e, por fim, desagregadas e passadas em peneira $\mathrm{ABNT} \mathrm{n}^{\circ} 325$ (diâmetro médio de partícula de $45 \mu \mathrm{m})$.

Os nanocompósitos de PET, contendo $1 \%$ em massa de argila modificada com os sais alquil amônio (A), alquil fosfônio (F) e a mistura de ambos os sais (A e F), foram preparadas em um misturador interno acoplado ao reômetro de torque Haake System 90 , operando com rotores do tipo roller a $260{ }^{\circ} \mathrm{C}$ e $60 \mathrm{rpm}$ por 10 minutos. Os híbridos de PET contendo as argilas AN, AP e MMT modificadas organicamente com os sais A e F foram codificados como PET/ANOA, PET/APOA, PET/MMTOA e PET/ANOF, PET/APOF, PET/MMTOF, respectivamente. Os híbridos preparados com a argila purificada modificada com a mistura dos sais A e F, foi nomeado como PET/APOA:F. Antes da mistura, o PET e as argilas organofílicas foram secos em estufa de secagem a 160 e $60{ }^{\circ} \mathrm{C}$ por 6 horas, respectivamente.

\section{Caracterização}

\section{Análise química}

A análise química das argilas não modificadas organicamente foi realizada pelo método semi-quantitativo, em forma de pastilha, sob atmosfera de hélio, em um espectrômetro de fluorescência de raios X Rigaku, modelo RIX 3000, equipado com tubo de Rh.

\section{Difratometria de Raios $X(D R X)$}

As medidas de DRX foram conduzidas à temperatura ambiente em um equipamento Shimadzu XDR-6000, utilizando radiação Cuk $\alpha(\lambda=1,5418 \AA$ ) , tensão de $40 \mathrm{kV}$, corrente de $30 \mathrm{~mA}$. As argilas organofílicas e os híbridos PET/argila organofílica foram examinados em um intervalo de $2 \theta$ entre 1,5 e $10^{\circ}$. A velocidade de varredura foi de $2 \% \mathrm{~min}$. As amostras de argila foram utilizadas na forma de pó com granulometria inferior a $45 \mu \mathrm{m}$, e os híbridos na forma de discos prensados com espessura média de $1,5 \mathrm{~mm}$, depois de preparadas no reômetro de torque Haake. Com esta técnica é possível confirmar a intercalação dos sais orgânicos nas galerias da argila pela expansão interlamelar basal da argila e investigar se um microcompósito ou um nanocompósito foi produzido ${ }^{[33]}$.

\section{Análise Termogravimétrica (TG)}

As análises de TG dos híbridos PET/argila organofílica foram conduzidas em aparelho TGA S1H da Shimadzu, sob atmosfera de ar a um fluxo de $50 \mathrm{~mL} / \mathrm{min}$, empregando uma quantidade de amostra em torno de $15 \mathrm{mg}$, taxa de aquecimento de $10{ }^{\circ} \mathrm{C} / \mathrm{min}$ e temperatura de 25 a $900{ }^{\circ} \mathrm{C}$.

\section{Calorimetria Exploratória Diferencial (DSC)}

As análises de DSC do PET puro e dos híbridos foram conduzidas em aparelho Perkim Elmer, modelo Pyris 6-DSC, operando a uma taxa de $10{ }^{\circ} \mathrm{C} / \mathrm{min}$, de 30 a $280{ }^{\circ} \mathrm{C}$ e de 280 a $30{ }^{\circ} \mathrm{C}$ sob atmosfera de nitrogênio a $20 \mathrm{~mL} / \mathrm{min}$, utilizando panela de alumínio. A 
quantidade de amostra empregada foi de aproximadamente $5 \mathrm{mg}$. Esta análise foi conduzida visando avaliar o efeito do tipo de argila e de modificador orgânico na velocidade de cristalização do PET.

\section{Microscopia Eletrônica de Transmissão (MET)}

A MET foi usada para avaliar a dispersão e o grau de intercalação e esfoliação da argila no polímero PET. As análises de MET foram realizadas em microscópio eletrônico de transmissão fabricado pela FEI, Tecnai 20, operando a uma voltagem de aceleração de 200 kV. As amostras analisadas foram prensadas na forma de discos com espessura média de $1,5 \mathrm{~mm}$, depois de preparadas no reômetro de torque Haake. As amostras foram preparadas através da redução de área pelo procedimento de "trimmer", em forma trapezoidal com uma área de aproximadamente $0,5 \mathrm{~mm}^{2}$. Os cortes das amostras foram realizados em um ultramicrótomo Leica, modelo EM UCG All Tech, usando uma faca de diamante, com velocidade de corte de $2 \mathrm{~mm} / \mathrm{s}$ e espessura em torno de $70 \mathrm{~nm}$. Os cortes foram colocados em grades de cobre de 300 mesh.

\section{Resultados e Discussão}

\section{Caracterização das argilas}

\section{Análise química}

Os dados de análise química das argilas não modificadas organicamente AN, AP e MMT estão reportados na Tabela 1. As bentonitas $\mathrm{AN}$ e AP apresentaram altos níveis de $\mathrm{SiO}_{2}$ e $\mathrm{Al}_{2} \mathrm{O}_{3}$, indicando que uma grande quantidade de montmorilonita está presente nestas bentonitas ${ }^{[18]}$. Estes percentuais são apenas um pouco inferiores aos da MMT comercial. A análise química mostra também a presença de óxidos metálicos como $\mathrm{Fe}_{2} \mathrm{O}_{3}, \mathrm{CaO}, \mathrm{MgO}$,

Tabela 1. Análise química das argilas não modificadas organicamente.

\begin{tabular}{|c|c|c|c|}
\hline Óxidos & $\begin{array}{l}\text { AN } \\
(\%)\end{array}$ & $\begin{array}{l}\text { AP } \\
(\%)\end{array}$ & $\begin{array}{c}\text { MMT } \\
(\%)\end{array}$ \\
\hline $\mathrm{SiO}_{2}$ & 41,88 & 37,21 & 49,94 \\
\hline $\mathrm{Al}_{2} \mathrm{O}_{3}$ & 10,53 & 9,11 & 13,89 \\
\hline $\mathrm{Fe}_{2} \mathrm{O}_{3}$ & 12,73 & 9,02 & 10,44 \\
\hline $\mathrm{FeO}$ & 1,44 & 4,06 & 4,95 \\
\hline $\mathrm{SO}_{3}$ & 4,09 & 8,06 & - \\
\hline $\mathrm{TiO}_{2}$ & 1,77 & 2,66 & 0,74 \\
\hline $\mathrm{K}_{2} \mathrm{O}$ & 0,73 & 1,26 & 0,15 \\
\hline $\mathrm{P}_{2} \mathrm{O}_{5}$ & 0,88 & 0,98 & 1,00 \\
\hline $\mathrm{Na}_{2} \mathrm{O}$ & 0,15 & 0,69 & 0,36 \\
\hline $\mathrm{MgO}$ & 0,85 & 0,63 & 0,66 \\
\hline $\mathrm{CaO}$ & 4,03 & 0,50 & 0,66 \\
\hline $\mathrm{ZrO}_{2}$ & 0,43 & 0,38 & 0,09 \\
\hline $\mathrm{ZnO}$ & 0,02 & 0,08 & 0,08 \\
\hline $\mathrm{MnO}$ & 0,04 & 0,06 & 0,03 \\
\hline $\mathrm{CuO}$ & - & 0,05 & 0,01 \\
\hline $\mathrm{Cl}$ & 0,48 & 0,03 & - \\
\hline $\mathrm{NiO}$ & 0,02 & 0,03 & 0,02 \\
\hline $\mathrm{SrO}$ & 0,10 & 0,03 & 0,01 \\
\hline $\mathrm{Y}_{2} \mathrm{O}_{3}$ & 0,02 & 0,03 & 0,03 \\
\hline $\mathrm{Ga}_{2} \mathrm{O}_{3}$ & 0,01 & 0,02 & 0,03 \\
\hline $\mathrm{Rb}_{2} \mathrm{O}$ & 0,01 & 0,01 & - \\
\hline Perda ao rubro & 19,61 & 24,68 & 16,35 \\
\hline Total & 99,84 & 99,56 & 99,46 \\
\hline $\mathrm{Fe}_{2} \mathrm{O}_{3} \mathrm{t}$ & 14,17 & 13,08 & 15,39 \\
\hline
\end{tabular}

$\mathrm{Na}_{2} \mathrm{O}, \mathrm{K}_{2} \mathrm{O}$ e os percentuais de perda ao rubro. Os percentuais dos óxidos metálicos e da perda ao rubrodas argilas AN e MMT foram semelhantes (Tabela 1). Por outro lado, a argila AP apresentou uma diferença em todos os percentuais referidos quando comparado com $\mathrm{a}$ argila AN. A argila AP apresentou um teor de $\mathrm{Fe}_{2} \mathrm{O}_{3}$ inferior ( 4\%) ao da argila $\mathrm{AN}$ e um teor de $\mathrm{FeO}$ superior $(\sim 3 \%)$. A razão para a diminuição no teor de $\mathrm{Fe}_{2} \mathrm{O}_{3}$ pode ser devido ao fato de que o $\mathrm{Fe}_{2} \mathrm{O}_{3}$ em condições favoráveis pode converter-se na sua forma solúvel, dissolvendo-se em contato com o meio oxidante (tratamento de purificação). Com relação ao aumento no teor de $\mathrm{FeO}$ a explicação pode ser a seguinte: o oxigênio existente na reação de purificação, por determinado tempo, pode reagir com o ferro da argila formando um composto insolúvel $(\mathrm{FeO})$, normalmente óxido. Observa-se ainda (Tabela 1) que a ordem quanto ao teor total de ferro $\left(\mathrm{Fe}_{2} \mathrm{O}_{3}+\mathrm{FeO}\right)$ para as três argilas é a seguinte: $\mathrm{MMT}>\mathrm{AN}>\mathrm{AP}$ indicando que a Cloisite ${ }^{\circledR} \mathrm{Na}^{+}$(MMT) tem maior quantidade de sítios catalíticos ativos do que as demais argilas, podendo, portanto, iniciar a degradação do polímero durante o processamento ${ }^{[13,17,25,34]}$.

A maior perda ao rubro apresentada pela argila AP pode ser atribuída à presença de resíduos insolúveis como carbonatos, sulfatos e fosfatos provenientes dos reagentes químicos empregados no processo de purificação, já que, de acordo com Souza Santos $(1989)^{[18]}$ a perda ao rubro é proveniente das perdas de água intercalada, de coordenação, de hidróxidos, componentes voláteis, carbonatos, sulfatos e fosfatos.

\section{Difratometria de raios $X$}

A difratometria de raios $X$ é a técnica mais utilizada para caracterizar híbridos de argilas com surfactantes. Esta técnica permite identificar os minerais existentes na amostra assim como avaliar a obtenção ou não de argilas organofílicas comparando o espaçamento basal $\left(\mathrm{d}_{001}\right)$ da argila modificada organicamente com o da argila não modificada ${ }^{[35]}$. Os difratogramas das argilas modificadas organicamente (Figura 1) mostram que os valores da distância interlamelar basal das argilas $\left(\mathrm{d}_{001}\right)$ AN $(1,34 \mathrm{~nm})$, $\operatorname{AP}(1,36 \mathrm{~nm})$ e MMT $(1,47 \mathrm{~nm})$ aumentaram após o processo de organofilização. Os aumentos foram de 43, 41 e 29\% para ANOA, APOA e MMTOA (Figura 1a) e de 71, 82 e 55\% para ANOF, APOF e MMTOF (Figura 1b), respectivamente. Estes dados confirmam a intercalação dos cátions orgânicos no espaçamento basal das argilas e, portanto, a obtenção de argilas organofílicas ${ }^{[36,37]}$.

As argilas modificadas com o sal F (Figura 1b) apresentaram estrutura do tipo pseudo-tricamada e valores de $\mathrm{d}_{001}$ superiores aos das argilas modificadas com o sal alquil amônio (A), o que reflete a formação de arranjos do tipo bicamada lateral para este último caso $^{[38]}$ (Figura 1a). Possivelmente a expansão entre as camadas da argila é mais influenciada pelo sal $\mathrm{F}$ do que pelo sal $\mathrm{A}$, uma vez que o sal $\mathrm{F}$ apresenta-se mais volumoso e com maior cadeia alquílica. Conforme reportado por Klapyta et al. (2001) ${ }^{[39]}$ e Yui et al. $(2002)^{[40]}$ o aumento no espaçamento basal de argilas organofílicas depende do comprimento das cadeias alquílicas e da densidade de empacotamento, além do arranjo conformacional da molécula do surfactante entre as camadas da argila. Resultados semelhantes foram reportados por Patel et al. (2007) ${ }^{[29]}$. Eles mostraram que a modificação orgânica de argilas com o sal brometo de tributil hexadecil fosfônio recobre eficientemente a superfície da argila, resultando na diminuição das forças de ligação entre as camadas da mesma e, favorecendo, portanto, num maior espaçamento basal.

A argila purificada modificada organicamente com a mistura dos sais A e F (Figura 1a) apresentou um espaçamento basal de 1,83 nm o que corresponde a um arranjo do tipo bicamada lateral. Comparando com a argila AP não modificada organicamente $\left(\mathrm{d}_{001}=1,36 \mathrm{~nm}\right) \mathrm{o}$ aumento foi de $34 \%$. Esta composição apresentou comportamento semelhante aos das argilas modificadas com o sal A. 


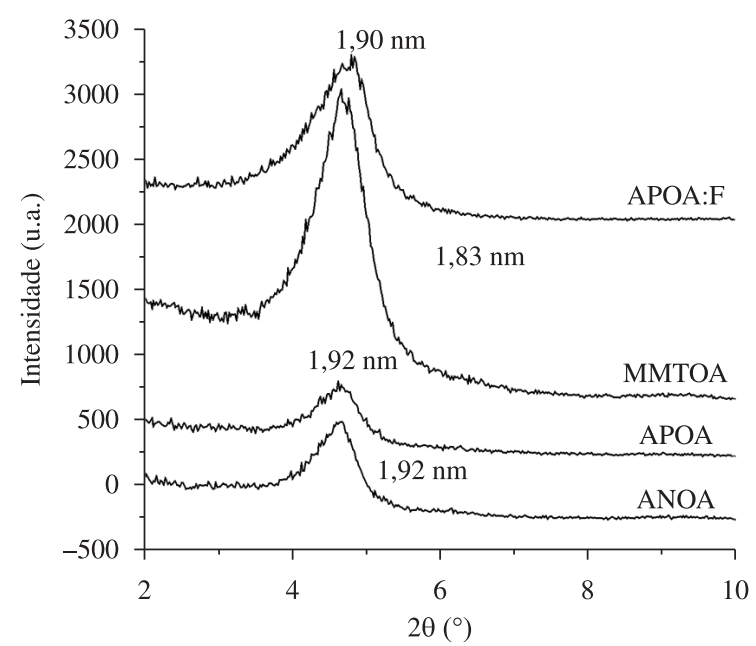

(a)

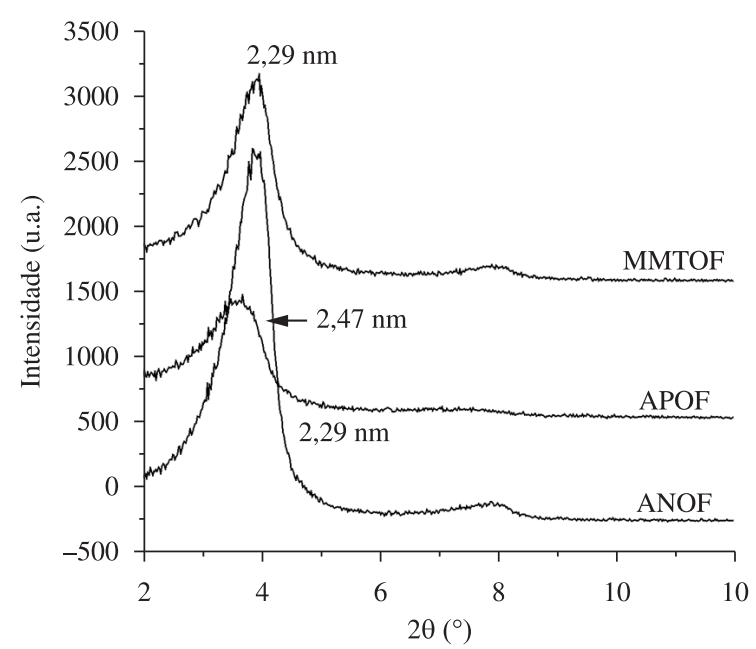

(b)

Figura 1. Difratogramas das argilas modificadas com o sal alquil amônio (A) e a mistura dos sais A e F (a) e com o sal alquil fosfônio (P) (b).

\section{Caracterização dos hibridos de PET}

\section{Difratometria de raios $X$}

Para investigar a estrutura dos híbridos de PET, as técnicas de difratometria de raios X (DRX) e microscopia eletrônica de transmissão (MET) foram utilizadas já que essas são ferramentas essenciais e indispensáveis para avaliar e identificar a intercalação/ esfoliação dos nanocompósitos poliméricos ${ }^{[41]}$.

Os difratogramas dos híbridos de PET, contendo $1 \%$ em massa de argila organofílica, estão apresentados na Figura 2. Verifica-se que o espaçamento basal $\left(\mathrm{d}_{001}\right)$ de todas as argilas modificadas com o sal A (Figura 1a) aumentou quando estas foram incorporadas na matriz PET (Figura 2a). Isto indica que cadeias do PET penetraram entre as camadas das argilas e que nanocompósitos intercalados foram obtidos. Verifica-se também que quando a argila APOA:F, argila organofilizada com a mistura dos sais A e F (Figura 1a), foi incorporada ao PET, o pico correspondente a reflexão basal (001) da argila não foi registrado no espectro (Figura 2a), sugerindo que as camadas de argila estão desordenadas na matriz de PET e que nanocompósitos com morfologia intercalada desordenada ou predominantemente esfoliada foram preparados. Para confirmar esta observação, o híbrido PET/APOA:F foi também caracterizado por microscopia eletrônica de transmissão (MET) e difratometria de raios $\mathrm{X}$ a baixos ângulos e os resultados estão mostrados na Figura 3.

Ao contrário do observado para as argilas organofilizadas com o sal A e com a mistura dos sais A e F, a incorporação das argilas organofilizadas com o sal $\mathrm{F}$ ao PET não resultou na formação de nanocompósitos, já que os valores do $\mathrm{d}_{001}$ das argilas organofilizadas com o referido sal (Figura 1b) permaneceram praticamente inalterados quando estas foram incorporadas a matriz polimérica (Figura 2b). A afinidade do cátion orgânico fósforo, presente no sal F, com os grupos terminais da cadeia de PET não é favorecida, podendo ser esta a razão para tal comportamento. Segundo Kawasumi et al. (1997) ${ }^{[42]}$, Cho \& Paul (2001 ${ }^{[43]}$ e Sánchez-Solís et al. (2004) $)^{[44]}$ o sucesso na dispersão, em escala nanométrica, dos silicatos em camadas dentro de matrizes poliméricas está associado com a presença de fortes interações entre a argila e as cadeias poliméricas. Portanto, além do espaçamento basal apresentado pela argila organofílica, a afinidade polímero/argila é essencial para se ter dispersão das argilas na matriz polimérica e, assim favorecer a obtenção de nanocompósitos.
Microscopia eletrônica de transmissão e difratometria de raios $X$ a baixos ângulos

A morfologia do híbrido PET/APOA:F, sugerida pela observação de DRX a altos ângulos, foi avaliada por microscopia eletrônica de transmissão (MET) e difratometria de raios $\mathrm{X}$ a baixos ângulos e os resultados obtidos estão apresentados nas Figuras $3 \mathrm{a}$ e 3b, respectivamente. De acordo com os dados de MET (Figura 3a), o híbrido PET/APOA:F exibe uma morfologia intercalada desordenada, conforme indicado pelos diferentes padrões de orientação e diferentes espaçamentos entre as camadas de argila (áreas escuras) na matriz polimérica (áreas claras). Com relação aos dados de difração de raios $\mathrm{X}$ a baixos ângulos (Figura 3b), observa-se que a intensidade do pico (001) da argila APOA:F no híbrido PET/APOA:F é significativamente inferior a do pico da argila APOA no híbrido PET/APOA. Isto indica que a morfologia do híbrido PET/APOA:F é do tipo intercalada desordenada, corroborando os dados de DRX a altos ângulos apresentados na Figura 2a.

Embora tenha sido reportado na literatura que argilas modificadas com fosfônio podem ser potencialmente úteis para a preparação de nanocompósitos poliméricos a base de $\operatorname{PET}^{[23,29,45-49]}$ já que a estabilidade térmica das mesmas é superior a $300{ }^{\circ} \mathrm{C}$, não foram encontrados estudos mostrando a obtenção de nanocompósitos de PET intercalados ou esfoliados, preparados com este tipo de argila. Neste trabalho observou-se que a utilização de argilas modificadas com o sal alquil fosfônio promoveu apenas a formação de um microcompósito e que a obtenção de um nanocompósito polimérico foi possível somente quando argilas modificadas com o alquil amônio e com a mistura dos sais alquil amônio e alquil fosfônio foram empregadas.

\section{Análise Termogravimétrica (TG)}

A Tabela 2 mostra as temperaturas de decomposição térmica, sob atmosfera de ar, do PET puro e dos híbridos PET/argila organofílica, correspondentes a 2,5;5,0 e $10,0 \%$ de perda de massa $\left(\mathrm{T}_{\mathrm{D} 2,5 \%}\right.$; $\mathrm{T}_{\mathrm{D} 5,0 \%}$ e $\mathrm{T}_{\mathrm{D} 10,0 \%}$ ), obtidas a partir das curvas termogravimétricas dos mesmos. Verifica-se que o tipo de argila e o tipo de modificador orgânico afetaram a estabilidade térmica do PET.

Quando a argila organofílica ANOA foi incorporada ao PET, as temperaturas de decomposição térmica $\mathrm{T}_{\mathrm{D} 2.5 \%} ; \mathrm{T}_{\mathrm{D} 5,0 \%}$ e $\mathrm{T}_{\mathrm{D} 10,0 \%}$ do híbrido PET/ANOA foram superiores as do PET puro. Por outro lado, quando as argilas organofílicas APOA e MMTOA foram 


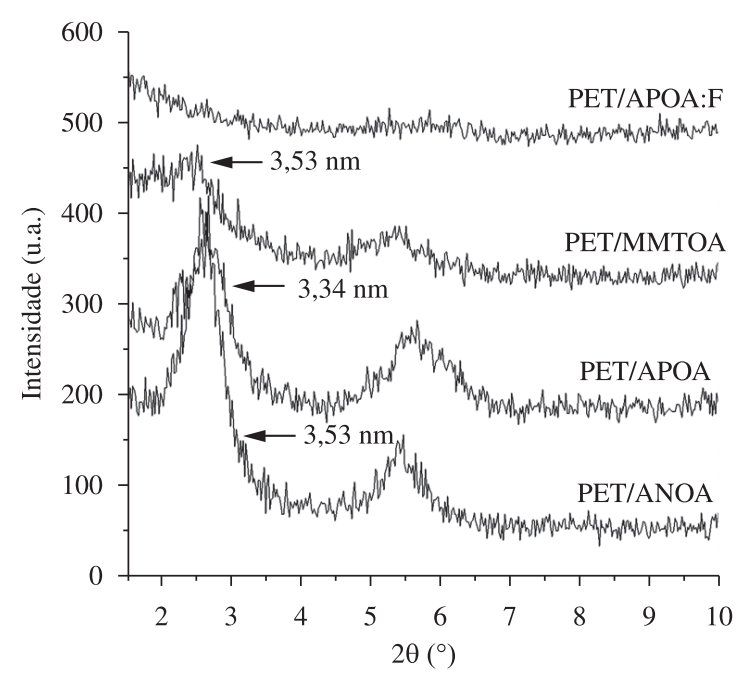

(a)

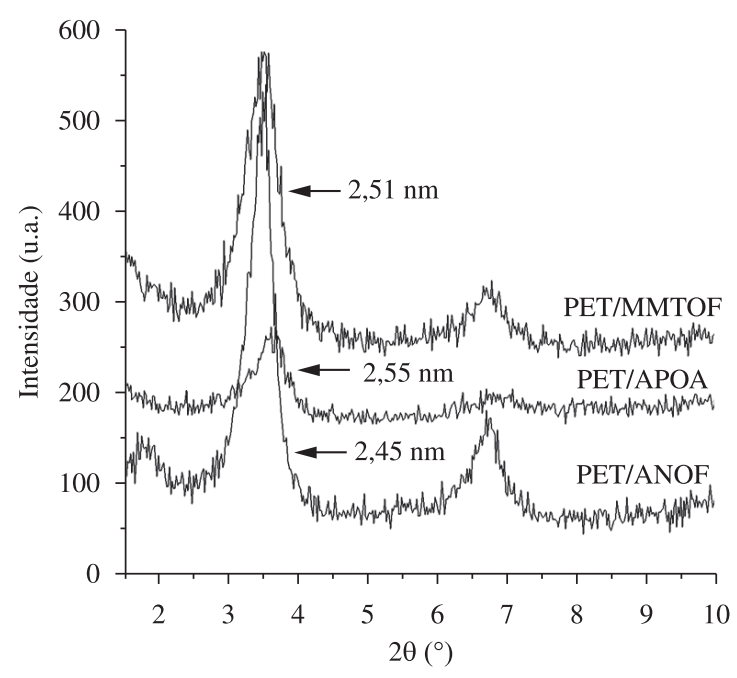

(b)

Figura 2. Difratogramas dos híbridos de PET contendo $1 \%$ em massa de argila modificada com os sais A e com a mistura dos sais A e F (argila purificada modificada) (a) e F (b).
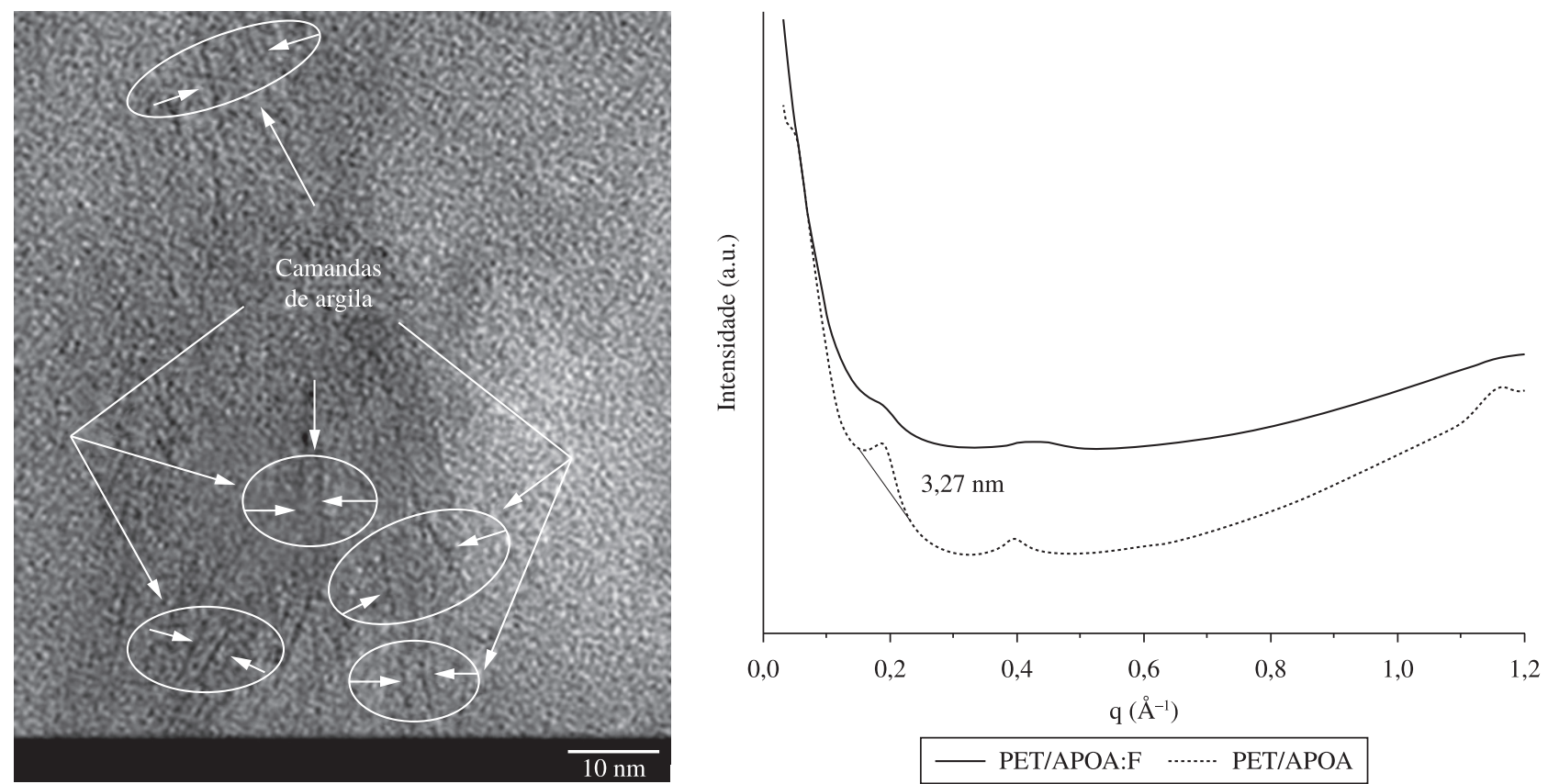

Figura 3. Imagem de MET do híbrido de PET contendo 1\% em massa da argila APOA:F (a) e difratometria de raios X a baixos ângulos dos híbridos PET/APOA:F e PET/APOA (b).

Tabela 2. Temperaturas de decomposição térmica do PET puro e dos híbridos de PET contendo $1 \%$ em massa de argila modificada com os sais alquil amônio (A), alquil fosfônio (F) e a mistura de ambos (A:F).

\begin{tabular}{cccc}
\hline Amostra & $\mathbf{T}_{\mathbf{D} 2,5 \%}\left({ }^{\circ} \mathbf{C}\right)$ & $\mathbf{T}_{\mathbf{D} 5, \mathbf{0} \%}\left({ }^{\circ} \mathbf{C}\right)$ & $\mathbf{T}_{\mathbf{D} 10,0 \%}\left({ }^{\circ} \mathbf{C}\right)$ \\
\hline PET Puro & 394,9 & 412,7 & 426,5 \\
PET/ANOA & 405,8 & 422,7 & 437,2 \\
PET/APOA & 391,4 & 411,1 & 426,8 \\
PET/MMTOA & 391,3 & 408,4 & 424,3 \\
PET/ANOF & 403,1 & 420,4 & 433,5 \\
PET/APOF & 399,2 & 417,4 & 433,7 \\
PET/MMTOF & 404,2 & 423,8 & 437,4 \\
PET/APOA:F & 398,5 & 416,7 & 432,8 \\
\hline
\end{tabular}

incorporadas, as temperaturas $\mathrm{T}_{\mathrm{D} 2.5 \%} ; \mathrm{T}_{\mathrm{D} 5.0 \%}$ e $\mathrm{T}_{\mathrm{D} 10,0 \%}$ dos híbridos PET/APOA e PET/MMTOA foram levemente inferiores as do PET puro. Isto indica que as nanopartículas de ANOA tiveram um melhor efeito na estabilização do PET. A razão para tal comportamento pode ser atribuída à maior ordem no empilhamento das camadas desta argila na matriz de PET, conforme evidenciado pelos dados de DRX (Figura 2a), onde o pico (001) da mesma (argila organofílica ANOA) no polímero (híbrido PET/ANOA) se mostrou mais intenso do que os picos (001) das argilas APOA e MMTOA no PET (híbridos PET/APOA e PET/MMTOA), sugerindo que a argila organofílica ANOA apresenta um arranjo intercalado mais ordenado dentro da matriz polimérica do que as argilas organofílicas APOA e MMTOA. 
Segundo Yoon et al. (2003) ${ }^{[50]}$ o melhor estado de dispersão da argila na matriz polimérica resulta na maior degradação do polímero devido à maior quantidade de superfície de argila e de amônio expostos a matriz, pois ambos resultam em reações de eliminação de Hofmann e produzem sítios ácidos Brønsted durante a preparação dos nanocompósitos, por fusão, acelerando a degradação do polímero ${ }^{[17]}$. Fornes et al. $(2003)^{[51]}$ reportaram que o melhor estado de dispersão da argila no polímero causou maior decomposição no polímero nylon-6. Tendência similar foi reportada por Matayabas \& Turner (2000) ${ }^{[52]}$ para nanocompósitos de PET/argila, onde o nível de degradação do PET aumentou à medida que a argila organofílica tornou-se mais esfoliada na matriz polimérica. Estes resultados estão de acordo com o observado neste estudo e corroboram a razão pela qual a estabilidade térmica do PET em presença das argilas organofílicas APOA e MMTOA foi menor do que em presença da argila organofílica ANOA.

Quando argilas modificadas com sal alquil fosfônio $(\mathrm{F})$ e a mistura de alquil amônio/alquil fosfônio (A:F) foram incorporadas ao PET, a estabilidade térmica de todos os híbridos foi superior a do PET puro, conforme pode ser visto pelos valores de $\mathrm{T}_{\mathrm{D} 2,5 \%}$; $\mathrm{T}_{\mathrm{D} 5,0 \%}$ e $\mathrm{T}_{\mathrm{D} 10,0 \%}$ apresentados na Tabela 2. Acredita-se que a maior estabilidade dos híbridos (PET/ANOF, PET/APOF, PET/MMTOF e PET/APOA:F) está associada com a maior ordem de empilhamento das argilas e a elevada estabilidade do alquil fosfônio. Com base nestes resultados fica evidenciado que a estabilidade térmica dos híbridos de PET/argila organofílica é determinada pelo tipo de surfactante e pela qualidade da dispersão da argila na matriz polimérica.

\section{Calorimetria exploratória diferencial}

Visando avaliar o efeito do tipo de argila e do tipo de modificador orgânico no processo de cristalização do PET, medidas de DSC foram conduzidas. A Figura 4 apresenta as curvas de cristalização, sob resfriamento a partir da fusão, a $10{ }^{\circ} \mathrm{C} / \mathrm{min}$, do PET puro e dos híbridos de PET contendo $1 \%$ em massa de argila organofilizada com os sais A, F e com a mistura de ambos os sais (A e F). Com exceção dos sistemas PET/ANOF e PET/APOA:F, que apresentaram temperatura de critalização $\left(\mathrm{T}_{c}\right)$ inferior e próxima a do PET puro, respectivamente, a $\mathrm{T}_{c}$ dos híbridos aumentou, em média de 11 a $18{ }^{\circ} \mathrm{C}$, em relação ao PET puro, devido, possivelmente, ao efeito da nucleação heterogênea das partículas

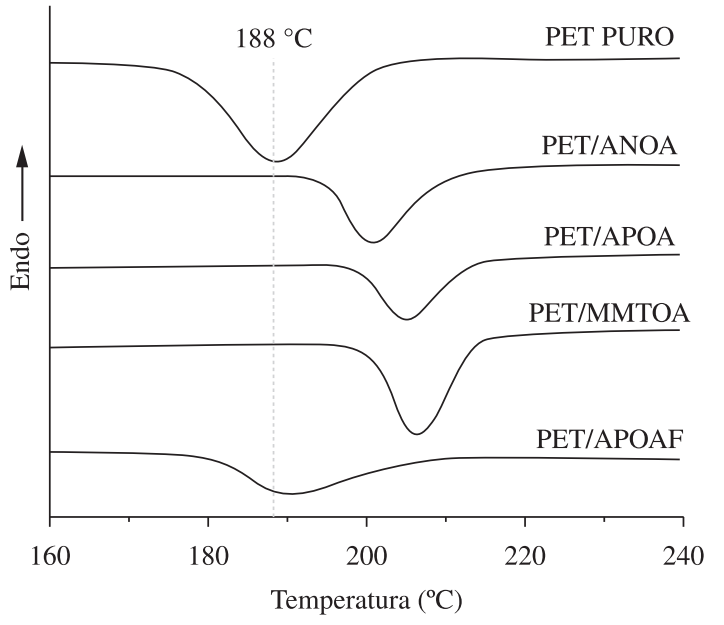

(a) de argila no processo de cristalização do PET. No estado fundido, os seguimentos das moléculas de PET podem interagir com a superfície da argila, originando núcleos de cristalização ${ }^{[12]}$. Outra possível razão para o aumento na $\mathrm{T}_{c}$ do $\mathrm{PET}$ com a incorporação de argilas organofílicas é que a carga inorgânica pode favorecer ao empilhamento ou ao ordenamento das cadeias de PET para o crescimento dos cristalitos ${ }^{[53]}$

Os valores de $\mathrm{T}_{\text {c }}$ para os híbridos variaram em função do tipo de argila e do tipo de modificador orgânico (Figura 4). Os valores de $\mathrm{T}_{\mathrm{c}}$ para PET/ANOA $\left(201{ }^{\circ} \mathrm{C}\right)$ e PET/ANOF $\left(183{ }^{\circ} \mathrm{C}\right)$ são bem diferentes e a atuação da argila AN como nucleante heterogêneo para o PET foi possível apenas quando a mesma foi organofilizada com amônio. A maior distância interplanar basal e menor ordem no empilhamento das camadas da argila ANOA na matriz de PET (menor intensidade do pico (001)), conforme mostrado na Figura 2, comparada com a argila ANOF, sugere que a área superficial das partículas de argila disponível para interações no PET/ANOA deve ter sido maior, resultando em um eficiente efeito nucleante. O mesmo comportamento foi observado para as argilas APO e MMT, ou seja, maiores valores de $T_{c}$ foram apresentados pelas argilas organofilizadas com o sal amônio (A), tendo em vista que a maior distância interplanar basal $\left(\mathrm{d}_{001}\right)$ e o arranjo mais desordenado apresentados pelas argilas modificadas com sal o A (conforme evidenciado pela menor intensidade do pico (001) (Figura 2), resultam em um efeito nucleante maior. Mas, porque a diferença entre a $\mathrm{T}_{c}$ do PET/APOA $\left(205{ }^{\circ} \mathrm{C}\right)$ e do PET/APOF $\left(202{ }^{\circ} \mathrm{C}\right)$ foi tão pequena? A resposta pode ser a seguinte: observando as intensidades dos picos (001) da argila APOA e da argila APOF, Figuras $2 \mathrm{a}$ e $2 \mathrm{~b}$, respectivamente, verifica-se que as mesma são semelhantes, embora as distâncias basais sejam diferentes, o que pode ter resultado em semelhança no tamanho da área superficial das partículas de argila disponível para interações com o polímero. Um outro questionamento é: porque a argila APOA:F não atuou eficientemente no aumento da temperatura de cristalização do PET já que a morfologia apresentada pelo híbrido PET/APOA:F foi intercalada desordenada tendendo a esfoliada, conforme dados mostrados nas Figuras 2 e 3 ?

É sabido que a eficiência de uma argila como nucleante heterogêneo para polímeros semi-cristalinos depende de vários fatores, dentre eles o estado de dispersão da argila na matriz e o estado de modificação da superfície são determinantes. Com base

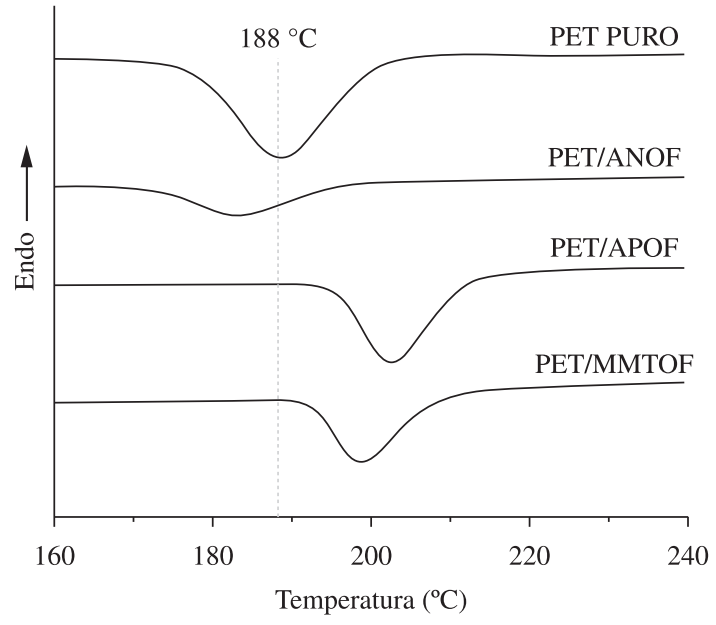

(b)

Figura 4. Curvas de DSC a uma taxa de resfriamento de $10{ }^{\circ} \mathrm{C} / \mathrm{min}$ : a) PET puro e híbridos de PET com argila organofilizada com sal amônio (A) e a mistura dos sais amônio e fosfônio (A:F) e b) PET puro e híbridos de PET com argila organofilizada com sal fosfônio (F). 


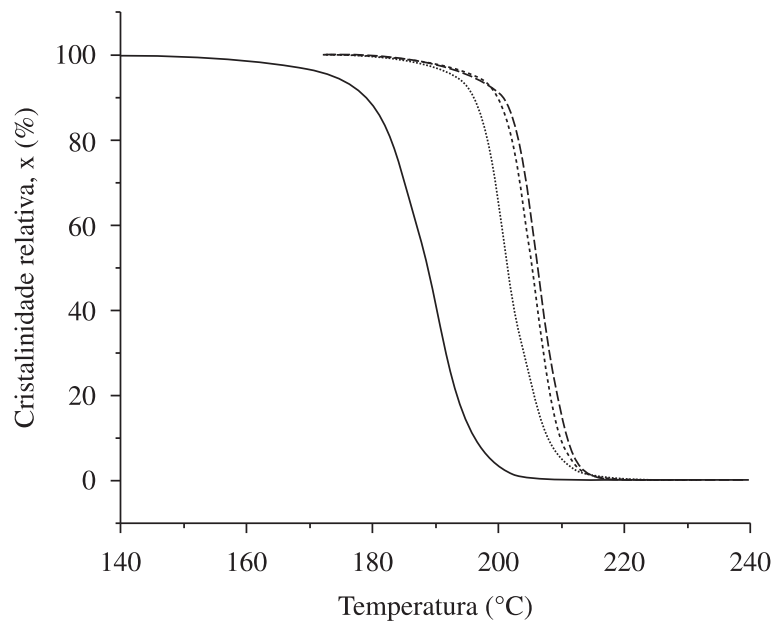

(a)

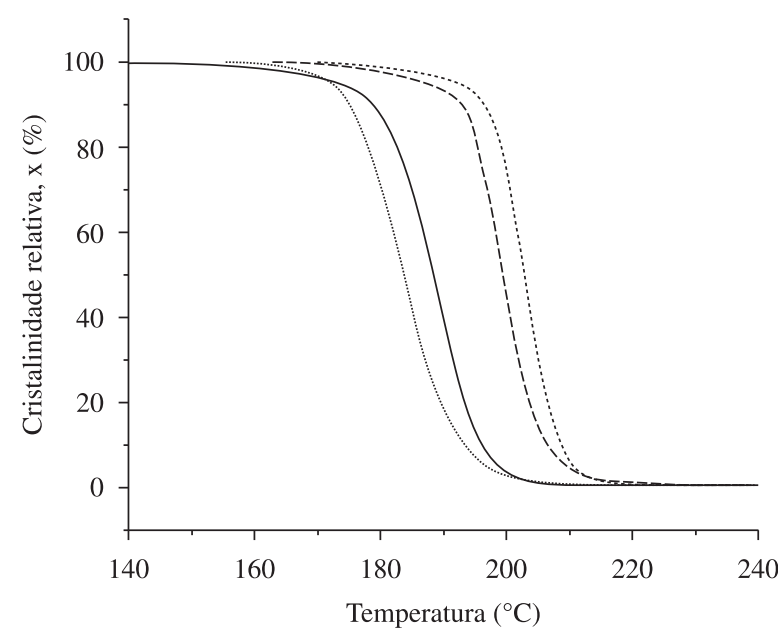

(b)

— PETPURO ……. PETANOA …. PETAPOA --- PETAMMTOA

Figura 5. Grau relativo de cristalinidade (x) com a temperatura sob taxa de resfriamento de $10^{\circ} \mathrm{C} / \mathrm{min}$ : a) PET puro e híbridos de PET com argilas organofilizadas com sal amônio (A) e b) PET puro e híbridos de PET com argilas organofilizadas com sal fosfônio (F).

nisto, as argilas devem ser bem dispersas na matriz polimérica, de forma a apresentar elevada área superficial, e devem também favorecer ao empilhamento e ao ordenamento de cadeias poliméricas, contribuindo desta forma para a nucleação e crescimento dos cristais. Entretanto, se a argila estiver dispersa dentro da matriz polimérica em escala nanométrica, onde os tamanhos das partículas de argila se encontram próximos aos das cadeias poliméricas, o molhamento das partículas de argila pelas cadeias poliméricas não é favorecido e neste caso a argila não atuará como agente de nucleante heterogêneo para o PET. A diminuição na velocidade de cristalização do PET com o aumento na dispersão das camadas de argila (estado esfoliado) no polímero também foi observado por Barber e colaboradores ${ }^{[54]}$.

A partir dos dados de cristalização não isotérmica obtidos por DSC, a cristalinidade relativa, como uma função da temperatura, do PET puro e dos híbridos de PET/argila organofílica, foi determinada usando a Equação 1:

$$
X_{t}=\int_{T_{0}}^{T}\left(d H_{c} / d T\right) / \int_{T_{0}}^{T \infty}\left(d H_{c} / d T\right) d T
$$

onde $\mathrm{T}_{\mathrm{o}}$ e $\mathrm{T}_{\infty}$ são as temperaturas de cristalização inicial e final, respectivamente e $d H_{c} / d T$ é o fluxo de calor.

A Figura 5 mostra que nos intervalos entre 10 e $90 \%$ de cristalinidade relativa, os híbridos de PET/argila organofílica, exceto o híbrido PET/ANOF, alcançaram estes percentuais em temperaturas mais altas do que o PET puro submetido às mesmas condições de processamento. Isto confirmando a atuação das argilas ANOA, APOA, MMTOA, APOF e MMTOF como nucleantes heterogêneos para o PET.

Embora as argilas ANOA, APOA, MMTOA, APOF e MMTOF possam ser usadas como nucleantes heterogêneos para o PET, as argilas ANOA, APOF e MMTOF são as mais indicadas para tal finalidade, pois apresentaram um melhor efeito na estabilização do PET, conforme mostrado anteriormente (Tabela 2). Com a adição de apenas $1 \%$ em massa de ANOA, APOF e MMTOF ao PET, a temperatura de cristalização do mesmo aumento de 188 para 201, 202 e $199{ }^{\circ} \mathrm{C}$, respectivamente. Este é um resultado relevante do ponto de vista industrial, pois o PET é um polímero cristalizável, mas com baixa velocidade de cristalização ${ }^{[53]}$ e para que este polímero seja usado na preparação de artefatos injetados, o aumento na velocidade de cristalização deste polímero é de grande importância. $\mathrm{O}$ aumento na velocidade de cristalização do PET garante uma solidificação mais rápida do polímero fundido sob resfriamento. Isto leva a uma redução do tempo de ciclo durante a moldagem por injeção e, propriedades tais como resistência a tração, temperatura de distorção térmica e dureza são aumentadas pela ação do agente nuclenate. Por causa da diminuição no tamanho dos esferulitos, as propriedades óticas, como transparência e brilho do polímero nucleado são também melhoradas ${ }^{[55]}$. Além destas vantagens, como a argila proveniente de empresa local (Bentonit União Nordeste/ Campina Grande/Paraíba), organofilizada sem purificação prévia (ANOA) ou purificada antes da organofilização (APOF), apresentou comportamento semelhante ao da montmorilonita comercial Cloisite ${ }^{\circledR} \mathrm{Na}^{+}$(MMT), fornecida pela Southern Clay Products/ EUA, organofilizada nas mesmas condições da ANOA e APOA (MMTOF), o uso destas (ANOA e APOF) pode ser uma alternativa atraente para aplicações do PET moldado por injeção onde o custo é um fator primordial.

\section{Conclusões}

O tipo de argila e o tipo de modificador orgânico afetaram a morfologia e a estabilidade térmica do PET. A incorporação das argilas organofilizadas com o sal fosfônio ao PET não resultou na formação de nanocompósitos. As argilas organofílicas ANOA, APOF e MMTOF, quando misturadas ao PET, provocaram aumento da sua estabilidade térmica além de atuarem como agentes nucleantes heterogêneos para o referido polímero, aumentando sua temperatura de cristalização. Este resultado é significativo do ponto de vista industrial, pois a baixa velocidade de cristalização do PET dificulta seu uso na preparação de artefatos injetados. Além disso, como as argilas bentoníticas fornecidas por empresa local apresentaram comportamento semelhante ao da montmorilonita importada, o uso dessa matéria-prima de menor custo, modificada com tecnologia nacional, pode ser uma alternativa atraente para aplicações do PET moldado por injeção onde o custo é um fator primordial. 


\section{Agradecimentos}

Os autores agradecem ao CNPq, a RENAMI e ao INAMI pelo apoio financeiro, à Braskem/BA pela doação do polímero PET, a Bentonit União Nordeste S/A pela doação da bentonita Argel 35.

\section{Referências Bibliográficas}

1. Mazumdar, S. K. - "Composites Manufacturing: Materials, Product and Process Engineering", New York (2002).

2. Tidjani, A. \& Wilkie, C. A. - Polym. Degrad. Stab., 74, p.33 (2001). http://dx.doi.org/10.1016/S0141-3910(01)00061-1

3. Ayres E.; Oréfice R. L. - Polímeros: Ciência e Tecnologia, 17, p.339 (2007).

4. Rodrigues, A. W.; Brasileiro, M. I.; Araújo, W. D.; Araújo, E. M.; Melo, T. J. A. - Polímeros, 17, p.219 (2007).

5. Brito, G. F.; Oliveira, A. D.; Araújo, E. M.; Melo, T. J. A.; Barbosa, R; Ito, E. N. - Polímeros, 18, p.170 (2008).

6. Da Paz, R. A.; Leite, A. M. D.; Araújo, E. M.; Melo, T. J. A.; Barbosa, R.; Ito, E. N. - Polímeros, 18, p.341 (2008).

7. Rodolfo Junior, A.; Mei, L. H. I. - Polímeros, 19, p.1 (2009).

8. Morales, A. R.; Da Cruz, C. V. M.; Peres, L.; Ito, E. N. - Polímeros, 20, p.39 (2010).

9. Silva, R. P.; Mauler, R. S.; Oliveira, R. V. B.; Salles, C. A. - Polímeros, 20, p.46 (2010).

10. Madaleno, L.; Schjødt-Thomsen, J.; Pinto, J. C. - Composites Sci. Technol., 70, p.804, 2010. http://dx.doi.org/10.1016/j. compscitech.2010.01.016

11. Costache, M. C.; Heidecker, M. J.; Manias, E. \& Wilkie, C. A. Polymers Adv. Technol., 17, p.764 (2006). http://dx.doi.org/10.1002/ pat.752

12. Calcagno, C. I. W.; Mariani, C. M.; Teixeira, S. R. \& Mauler, R. S. - Polymer, 48, p.966 (2007). http://dx.doi.org/10.1016/j. polymer.2006.12.044

13. Xu, X.; Ding, Y.; Qian, Z.; Wang, F.; Wen, B.; Zhou, H.; Zhang, S. \& Yang, M. - Polymer Degrad. Stab., 94, p.113 (2009). http://dx.doi. org/10.1016/j.polymdegradstab.2008.09.009

14. González-Vidal, N.; Munõz-Guerra, S.; Ilarduya, A. M.; Benali, S.; Peeterbroeck, S. \& Dubois, P. - Eur. Polymer J., 46, p.156 (2010).

15. Ramos Filho, F. G.; Melo, T. J. A.; Rabello, M. S. \& Silva, S. M. L. Polym. Degrad. and Stab., 89, p.383 (2005).

16. Silva, S.; Araújo, P. E.; Ferreira, K. M.; Canedo, E.; Carvalho, L. \& Raposo, C. M. - Polymer Eng. Sci., 49, p.1696 (2009). http://dx.doi. org/10.1002/pen.21399

17. Leite, I. F.; Soares, A. P. S.; Carvalho, L. H.; Raposo, C. M. O.; Malta, O. M. L. \& Silva, S. M. L. - J. Thermal Anal. Calorim., 100, p.563 (2010). http://dx.doi.org/10.1007/s10973-009-0265-3

18. Souza Santos, P. - "Ciência e Tecnologia de Argilas", $2^{\text {nd }}$ ed., Edgard Blucher Ltda, São Paulo (1989).

19. Utracki, L. A. - "Clay-Containing Polymeric Nanocomposites", $1^{\text {nd }}$ ed., Rapra Technology Limited, United Kingdom (2004).

20. Velasco, J. I.; Ardanuy, M.; Miralles, L.; Ortiz, S.; Maspoch, M. L.; Sánchez-Soto, M. \& Santana, O. - Macromol. Symp., 221, p.63 (2005). http://dx.doi.org/10.1002/masy.200550307

21. Vaia, R. A.; Rachel, K. T. \& Giannelis, E. P. - Chem. Mater., 6, p.1017 (1994). http://dx.doi.org/10.1021/cm00043a025

22. Hasegawa, N.; Kawasumi, M.; Kato, M.; Usuki, A.; Okada, A. J. Appl. Polymer Sci., 67, p.87 (1998). http://dx.doi.org/10.1002/(SICI)10974628(19980103)67:1\%3C87::AID-APP10\%3E3.0.CO;2-2
23. Xie, W.; Xie, R.; Pan, W-P.; Hunter, D.; Koene, B. \& Tan, L-S.; Vaia, R. - Chem. Mater., 14, p.4837 (2002). http://dx.doi.org/10.1021/ cm020705y

24. Avalos, F.; Ortiz, J. C.; Zitzumbo, R.; López-Manchado, M. A.; Verdejo, R. \& Arroyo, M. - Europ. Polymer J., 44, p.3108 (2008). http://dx.doi.org/10.1016/j.eurpolymj.2008.07.020

25. Xie, W.; Gao, Z.; Liu, K.; Pan, W.; Vaia, R.; Hunter, D. \& Singh, A. Thermochim. Acta, 367-368, p.339 (2001). http://dx.doi.org/10.1016/ S0040-6031(00)00690-0

26. Stoeffler, K.; Lafleur,P.G. \& Denault, J.-Polym. Degrad.Stab.,93,p. 1332 (2008). http://dx.doi.org/10.1016/j.polymdegradstab.2008.03.029

27. Shah, R. K. \& Paul, D. R. - Polymer, 47, p.4075 (2006). http://dx.doi. org/10.1016/j.polymer.2006.02.031

28. Chigwada, G.; Wang, D.; \& Wilkie, C. A. - Appl. Clay Sci., 91, p.848 (2006).

29. Patel, H. A.; Somani, R. S.; Bajaj, H. C. \& Jasra, R. V. - Appl. Clay Sci., 35, p.194 (2007). http://dx.doi.org/10.1016/j.clay.2006.09.012

30. Davis, C. H.; Mathias, L. J.; Gilman, J. W.; Schiraldi, D. A.; Shields, J. R.; Trulove, P.; Sutto, T. E. \& Delong, H. C. - J. Polymer Sci.: Part B: Polymer Phys., 40, p.2661 (2002). http://dx.doi.org/10.1002/ polb. 10331

31. Phelps, G. W. \& Harris, D. L. - Amer. Ceram. Soc. Bull., 47, p.1146 (1968).

32. Araújo, P. E. R.; Silva, S. A.; Raposo, C. M. O.; Silva, S. M. L. "Poly(ethylene terephthalate)/PET/layered silicate nanocomposites: effect of bentonite purification on morphology/behaviour relationship". in: Proceedings of the $23^{\circ}$ Annual Meeting - The Polymer Processing Society (PPS), Salvador-BA, maio (2007).

33. Ray, S. S. \& Okamoto, M. - Progress in Polymer Sci., 28, p.1539 (2003).

34. Qin, H.; Zhang, S.; Liu, H.; Xie, S.; Yang, M. \& Shen, D. - Polymer, 46, p.3149 (2005). http://dx.doi.org/10.1016/j.polymer.2005.01.087

35. Paiva, L. B.; Morales, A. R. \& Díaz, F. R. V. - Appl. Clay Sci., 42, p.8 (2008).

36. Kornmann, X. - Polymer, 42, p.1303 (2001). http://dx.doi.org/10.1016/ S0032-3861(00)00346-3

37. Rodríguez-Sarmiento; D. C. \& Pinzón-Bello; J. A. - Appl. Clay Sci., 18, p.173 (2001).

38. Calderon, J. U.; Lennox, B. \& Kamal, M. R. - Appl. Clay Sci., 40, p.90 (2008). http://dx.doi.org/10.1016/j.clay.2007.08.004

39. Klapyta, Z.; Fujita, T. \& Iyi, N. - Appl. Clay Sci., 19, p.5 (2001). http:// dx.doi.org/10.1016/S0169-1317(01)00059-X

40. Yui, T.; Yoshida, H.; Tachibana, H.; Tryk, D. A. \& Inoue, H. - Langmuir, 18, p.891 (2002). http://dx.doi.org/10.1021/la011297x

41. Barbosa, R.; Araújo, E. M.; Maia, L. F.; Pereira, O. D.; Melo, T. J. A. \& Ito, E. N. - Polímeros, 16, p.246 (2006).

42. Kawasumi, M.; Hasegawa, N.; Kato, M.; Usuki, A. \& Okada, A. - Macromolecules, 30, p.6333 (1997). http://dx.doi.org/10.1021/ ma961786h

43. Cho, J. \& Paul, D. R. - Polymer, 42, p.1083 (2001). http://dx.doi. org/10.1016/S0032-3861(00)00380-3

44. Sánchez-Solís, A.; Romero-Ibarra, I.; Estrada, M. R.; Celderas, F. \& Manero, O. - Polymer Eng. Sci., 44, p.1094 (2004).

45. Chang, J., Seo, B. \& Hwang, D. - Polymer, 43, p.2969 (2002). http:// dx.doi.org/10.1016/S0032-3861(02)00125-8

46. Fornes, T. D.; Yoon, P. J.; Keskkula, H. \& Paul, D. R. - Polymer, 43, p.5915 (2002). http://dx.doi.org/10.1016/S0032-3861(02)00400-7 
47. Yuan, X.; Li, C.; Guan, G.; Xiao, Y. \& Zhang, D. - Polym. Degrad. Stab., 93, p.466 (2008). http://dx.doi.org/10.1016/j. polymdegradstab.2007.11.010

48. Hedley, C. B.; Yuan, G. \& Theng, B. K. G. - Appl. Clay Sci., 35, p.180 (2007). http://dx.doi.org/10.1016/j.clay.2006.09.005

49. Avalos, F.; Ortiz, J. C.; Zitzumbo, R.; López-Manchado, M. A.; Verdejo, R. \& Arroyo, M. - Appl. Clay Sci., 43, p. 27 (2009). http:// dx.doi.org/10.1016/j.clay.2008.07.008

50. Yoon, P. J.; Hunter, D. L.; Paul, D. R. - Polymer, 44, 18, p.5341. http:// dx.doi.org/10.1016/S0032-3861(03)00523-8

51. Fornes, T. D.; Yoon, P. J. \& Paul, D. R. - Polymer, 44, 24, p.7545 (2003). http://dx.doi.org/10.1016/j.polymer.2003.09.034
52. Matayabas, J. J.; Turner, S. - "Polymer-clay nanocomposites", John Wiley \& Sons Ltda, New York (2000).

53. Wang, Y.; Gao, J.; Ma, Y. \& Agarwal, U. S. - Composites: Part B, 37, p.399 (2006). http://dx.doi.org/10.1016/j.compositesb.2006.02.014

54. Barber, G. D.; Calhoun, B. H. \& Moore, R. B. - Polymer, 46, p.6706 (2005). http://dx.doi.org/10.1016/j.polymer.2005.05.024

55. Zweifel, H. - "Plastics Additives Handbook", Hanser Publishers, Munich (2001).

Enviado: $30 / 03 / 10$

Reenviado: $20 / 10 / 10$

Aceito: $10 / 11 / 10$

DOI: $10.1590 / \mathrm{S} 0104-14282011005000035$ 